\title{
EL ROL DEL ANÁLISIS DE GÉNERO EN LA REDUCCIÓN DE LOS SESGOS ALGORÍTMICOS
}

\author{
El análisis recogido en este artículo estudia el papel del análisis de género en combatir \\ los sesgos algorítmicos derivados de la falta de inclusión y visibilidad de la mujer tanto \\ en los macrodatos (big data) como en los algoritmos de inteligencia artificial (IA). Al \\ fin de analizar esta problemática, se estima el número de mujeres matriculadas en áreas \\ de estudios relacionados con la informática, la ingeniería y la ciencia de datos y se \\ analizan las barreras a la interdisciplinariedad que pueden facilitar la difusión de sesgos \\ algorítmicos y limitar el progreso de la economía digital.
}

\section{The role of gender analysis in the reduction of algorithmic biases}

The aim of this paper is to study the role that gender analysis may play to prevent algorithmic biases. These biases are caused by the lack of inclusion and visibility of women in both big data and in Artificial Intelligence algorithms. To tackle this issue, this article offers an estimation of the number of female students enrolled in fields related to computer science, engineering and data science and an analysis of existing barriers to interdisciplinary collaborations that can facilitate the proliferation of algorithmic biases and can hinder the progress of the digital economy.

Palabras clave: género, innovación, inteligencia artificial, big data, sesgos algorítmicos.

Keywords: gender studies, innovation, artificial intelligence, big data, algorithmic bias.

JEL: 032 .

\section{Introducción}

En España, así como en muchos otros países de la Unión Europea (UE), se evidencia la necesidad de alcanzar una representación equilibrada de hombres y mujeres en los distintos ámbitos económicos y

\footnotetext{
* Investigadora Juan de la Cierva - Incorporación (IJC2019-041898-I), Directora Científica e IP por el CSIC del proyecto H2020 TRESCA Trustworthy, Reliable and Engaging Scientific Communication Approaches (GA núm. 872855).

Instituto de Políticas y Bienes Públicos (IPP).

Centro de Ciencias Humanas y Sociales (CCHS).

Consejo Superior de Investigaciones Científicas (CSIC).

Contacto: sara.degli.esposti@csic.es

Versión de junio de 2021.

https://doi.org/10.32796/ice.2021.921.7265
}

sociales relacionados con el desarrollo del ecosistema digital. Según el Ministerio de Ciencia e Innovación, el impulso de la igualdad de género en el sistema de ciencia e innovación, además de ser un principio básico del derecho ${ }^{1}$, es garantía de excelencia en el sistema nacional de investigación, desarrollo e innovación $(\mathrm{I}+\mathrm{D}+\mathrm{i})$. La presencia equilibrada de hombres y mujeres contribuye -entre otros indicadores - a determinar el nivel de desarrollo de un país. El Índice

\footnotetext{
Hay que recordar al respecto: la Ley Orgánica 3/2007, de 22 de marzo, para la igualdad efectiva de mujeres y hombres («BOE» núm. 71, de 23 de marzo de 2007); el Real Decreto-ley 6/2019, de 1 de marzo, de medidas urgentes para garantía de la igualdad de trato y de oportunidades entre mujeres y hombres en el empleo y la ocupación («BOE» núm. 57, de 7 de marzo de 2019).
} 
de Igualdad de Género 2020 (calculado en base a datos de 2018) indica que España se sitúa 4 puntos por encima de la media de la Unión Europea (UE), con 72 puntos sobre 100 (EIGE, 2020), en términos de igualdad.

La inteligencia artificial (IA) y el big data —es decir, las tecnologías de gestión y análisis de macrodatosson consideradas las piedras angulares que sustentan la economía digital o la también denominada «economía de las plataformas» (Srnicek, 2017) o «economía de la vigilancia» (Zuboff, 2015). La creación de un ecosistema fiable que permita el desarrollo seguro de la IA de acuerdo con los valores y derechos de los ciudadanos de la UE, es un área estratégica prioritaria de la UE. En febrero de 2020, la Comisión Europea (CE) presentó el «Libro Blanco sobre la inteligencia artificial - un enfoque europeo orientado a la excelencia y la confianza ${ }^{2}$ con una previsión de inversiones por valor de 20.000 millones de euros al año durante la siguiente década. En asociación con la «Ley de Servicios Digitales» (Digital Services Act) y la «Ley de Mercados Digitales» (Digital Markets Act), la UE busca el liderazgo en el ámbito digital y la conservación de su soberanía tecnológica a pesar de su dependencia de empresas tecnológicas de Estados Unidos y China (Serrano et al., 2021).

La «Estrategia Española para la $\mid A$ », en línea con el «Plan Coordinado de la Inteligencia Artificial» (European Commission, 2018), identifica las siguientes prioridades: la construcción de un ecosistema de datos digitales y de tecnologías de macrodatos; la creación de estructuras organizativas para el desarrollo y la evaluación de la IA; la transferencia de conocimiento de la investigación científica a la industria; la identificación de áreas de aplicación de la IA con alto impacto social y económico; el fomento de la educación y la formación en IA a través de programas multidisciplinares y la evaluación de los aspectos éticos de la IA.

\footnotetext{
2 https://op.europa.eu/es/publication-detail/-/publication/ac957f13-53c611ea-aece-01aa75ed71a1
}

En contradicción con estos objetivos, en España desde el año 2009 se han venido desplomando tanto los presupuestos asignados a I+D+i, como su aplicación efectiva. Además, la I+D+i se convierte en el año 2019 en una de las partidas que menor tasa de ejecución presupuestaria registra (por debajo del $70 \%$ ) entre todas las políticas del sector público estatal, solo por detrás de "Comercio, Turismo y Pymes» $(42,5 \%)$ y en evidente contraposición con las políticas de «Pensiones, Defensa, Justicia» y «Seguridad Ciudadana», las que muestran tasas de ejecución por encima del $90 \%$ de su presupuesto (Fundación COTEC, 2021).

Dentro de este contexto, el presente artículo analiza el problema de la limitada presencia de mujeres en los colectivos que lideran el desarrollo de aquellas tecnologías habilitadoras a la base del ecosistema digital, es decir el big data y la IA. La falta de diversidad y de participación de los distintos grupos sociales que conforman la sociedad, entre ellos el de las mujeres, conlleva el riesgo de reproducir prejuicios y dinámicas de discriminación de género y puede, además, producir un empobrecimiento creativo e imaginativo de los sectores involucrados en el desarrollo de productos y servicios informáticos. Por último, la falta de diversidad de los equipos que diseñan y desarrollan tecnologías digitales puede impedir que haya personas capaces de hablar y conectar con las nuevas generaciones, mejorando sus conocimientos y nivel de concienciación sobre el abuso de los dispositivos digitales y sobre la dependencia y las vulnerabilidades generadas por la proliferación de estas tecnologías.

\section{El papel de la IA y del big data en la economía del dato}

En términos económicos, los macrodatos o big data indican una nueva forma de competición de mercado basada en la automatización del análisis de conjuntos de datos medidos en el orden de terabytes. Este tipo de análisis permite mejorar la eficiencia 
de procesos organizativos, personalizar productos y ofertas comerciales o identificar comportamiento fraudulento. El big data representa una fuente de ventaja competitiva y un nuevo estadio de lo que en los años noventa se solía llamar Business Intelligence y que hoy podríamos llamar big data analytics, es decir, la aplicación de la IA específica (en inglés, narrow $A I$ ) a los macrodatos.

Con IA específica nos referimos a sistemas de IA que están diseñados para manejar tareas bien definidas. Bajo este paraguas encontramos los algoritmos de aprendizaje automático (en inglés, machine learning) $\mathrm{y}$, dentro de este mundo, el aprendizaje profundo (en inglés, deep learning). Campos de estudios y áreas de aplicación concretas provienen del ámbito del procesamiento del lenguaje natural o PLN (en inglés, Natural Language Processing) o del reconocimiento automático de imágenes en el área de visión artificial (en inglés, computer vision). Estas tecnologías habilitadoras son consideradas las piedras angulares que sustentan el ecosistema digital y su modelo económico.

A pesar de que estos términos nos puedan sonar algo exóticos, sus aplicaciones nos rodean tanto en casa como en el trabajo. De hecho, podemos afirmar que los españoles están sumergidos en el ecosistema del big data y contribuyen con sus datos a la industria del marketing digital, que es la que financia gran parte de las plataformas de uso diario - como, por ejemplo, Facebook, Instagram, YouTube, o Google Search-entre otras. Sin embargo, según el «Índice de Economía y Sociedad Digital» (European Commission, 2020a), el nivel de alfabetización digital de la población es limitado y la mayoría desconoce la existencia o el funcionamiento de muchas de las tecnologías habilitadoras que permiten a los dispositivos inteligentes funcionar. No solamente se desconoce la tecnología, sino que también falta conocimiento de los términos contractuales de la mayoría de estos servicios.

La sencillez y rapidez con la que podemos abrir una nueva cuenta de usuario o descargar una nueva aplicación cumplen con un influyente dictamen en el mundo del diseño de sistemas cibernéticos: «las tecnologías más profundas son las que desaparecen» (Weiser, 1991, p. 94). La normalización -y consecuente invisibilización - de la tecnología en lo cotidiano es la medida más evidente de su aceptación y de su consecuente éxito comercial. Veremos en el siguiente apartado como este principio se aplica tanto a la tecnología como a las normas sociales, generando creencias y prejuicios que pueden penetrar en nuestros tejidos más profundos y que pueden volver a manifestarse de forma inesperada generando sesgos algorítmicos y de automatización. La influencia de las normas sociales y culturales sobre el desarrollo tecnológico ha generado un consenso en las áreas de estudio más concernidas con las implicaciones sociales y morales de la IA a hablar de «sistemas sociotécnicos», pues son sistemas donde tecnología y sociedad interactúan constantemente en un proceso llamado de coproducción (Jasanoff, 2004). El creciente uso de algoritmos para automatizar la toma de decisiones en varios ámbitos suscita una profunda preocupación por el riesgo de que se produzcan errores a gran escala que perjudiquen la vida de muchas personas (O'Neil, 2017). Los algoritmos plantean nuevos retos en ámbitos que, aún se rijan por protecciones legales diseñadas para evitar la discriminación ${ }^{3}$, ven su funcionamiento y formas de operar completamente revolucionados por la digitalización.

\footnotetext{
3 Entre las protecciones legales contra la discriminación por temas de género en la Unión Europea cabe recordar: i) la Directiva 2000/78/CE del Consejo, de 27 de noviembre de 2000 , relativa al establecimiento de un marco general para la igualdad de trato en el empleo y la ocupación; ii) la Directiva del Consejo 2004/113/CE, de 13 de diciembre de 2004, por la que se aplica el principio de igualdad de trato entre hombres y mujeres al acceso a bienes y servicios y su suministro; iii) la Directiva 2006/54/CE del Parlamento Europeo y del Consejo, de 5 de julio de 2006, relativa a la aplicación del principio de igualdad de oportunidades e igualdad de trato entre hombres y mujeres en asuntos de empleo y ocupación (refundición); y iv) la Directiva 2012/29/UE del Parlamento Europeo y del Consejo, de 25 de octubre de 2012, por la que se establecen normas mínimas sobre los derechos, el apoyo y la protección de las víctimas de delitos, y por la que se sustituye la Decisión marco 2001/220/JAI del Consejo.
} 
3. Viejos prejuicios y nuevos sesgos algorítmicos

Así como el rosa sigue siendo el color más utilizado en la confección de prendas y ajuar para las niñas, sigue existiendo una tendencia a dar una educación diferenciada a los niños y a las niñas en función de su sexo, perpetuando un sistema normativo incrustado en los roles de género. Sin entrar en el debate teórico de si estos roles de género ${ }^{4}$ sirven dinámicas funcionalistas de reproducción social o dinámicas de biopoder y de dominio de un grupo sobre otro grupo social, la perspectiva teórica que propone abarcar estos temas como «sistemas sociotécnicos» nos ayuda a evidenciar el carácter histórico y culturalmente situado del desarrollo tecnológico y el rol que juegan las normas de género en este desarrollo. Para entender la relación entre desarrollo tecnológico y normas sociales es suficiente pensar en el papel que ha jugado el marketing a partir de la Segunda Guerra Mundial, en cambiar nuestras percepciones de distintas cuestiones morales y estéticas en el proceso de asimilación de las normas de la economía del consumo de masa.

Volvemos al ejemplo del color rosa para las niñas y del azul para los niños. En 1927, la revista Time publicó una tabla en la que se mostraban los colores apropiados para el sexo de las niñas y los niños según las principales tiendas de Estados Unidos (EE UU). Antes de que los colores pastel fueran populares para los bebés, la mayoría de los padres vestían a sus hijos de blanco hasta que cumplían los seis años. Los baby boomers de la década de los cuarenta fueron los primeros estadounidenses en vestirse con ropa de colores específicos para su sexo (Paoletti, 2012). A pesar de que no haya pruebas de que estas preferencias de colores dependan de factores biológicos relacionados con el sexo de los niños (Del Giudice, 2017), hoy en

\footnotetext{
4 Sexólogos y psicólogos americanos introdujeron la palabra género en los años cincuenta, con la intención de distinguir el sexo anatómico del género social.
}

día nos parece apropiado regalar prendas rosas a los padres de una recién nacida bebé; esto a pesar de las críticas hechas por escritoras como Adela Turin ya en los años setenta. Exactamente como en el caso de la tecnología, cuanto más consuetudinaria es una norma mayor será su aceptación y, como consecuencia, su invisibilidad; una vez que se haya convertido en sentido común, la norma, con todo lo inofensiva que sea, entra a formar parte del conjunto de puntos ciegos (en inglés, blindspots) de una cultura.

Estos puntos ciegos son las celdas vacías y las variables omitidas en los macrodatos que hoy en día usan los programadores para entrenar sus algoritmos. El tema de que los macrodatos nos permitan ver aspectos de nuestra sociedad y, al mismo tiempo, no nos permitan ver otros aspectos es parte de un creciente debate científico en los que destacan mujeres como Meredith Whittaker y Kate Crawford, fundadoras del instituto de investigación Al Now en la Universidad de Nueva York (Whittaker et al., 2018). El tipo de investigación interdisciplinaria que se lleva a cabo en este instituto pretende ayudar a garantizar que los sistemas de IA incorporen principios de equidad, transparencia y rendición de cuentas (en inglés, accountability).

El enfoque adoptado por el AI Now presenta la cuestión de la problemática de género dentro del más amplio conjunto de problemas relacionados con equidad y datos digitales (Dencik et al., 2019). Desde esta perspectiva, la crisis de diversidad que se vive en el sector de la IA en cuanto a género y etnia se puede considerar tanto un síntoma como una causa de problemas técnicos que se están detectando en los sistemas y que se suelen llamar «sesgos algorítmicos» (West et al., 2019).

\section{Razones de por qué el desequilibrio de género en la I+D+i de IA es un problema}

La verdadera fuerza del pensamiento feminista no se encuentra en la denuncia de los privilegios del otro grupo social, en este caso «los hombres», sino en la elaboración de un concepto de ser humano que 
reconcilie, incluya y establezca diálogos entre experiencias existenciales muy diversas. A partir de este concepto inclusivo de ser humano reivindicaremos que tanto los programadores como las máquinas cumplan con principios de inclusión, no discriminación, equidad, trasparencia y rendición de cuentas. Esta idea amplia e inclusiva de persona nos permite no excluir a nadie a la hora de plantear cualquier cuestión relacionada con la equidad de oportunidades y condiciones. Desde esta perspectiva, es preciso discutir y argumentar el por qué es problemático el desequilibrio de género en la I+D+i en IA.

Entre las muchas teorías que abarcan el tema de las relaciones de género y su impacto sobre la economía y la sociedad, aquí adoptaremos dos perspectivas específicas que provienen de la teoría de las organizaciones y de la ciencia de la administración. La primera teoría habla de las ventajas -en términos de resultados o performance - de construir equipos de trabajo diversos, es decir, multiétnicos, tanto con hombres como con mujeres, con trayectorias profesionales internacionales, que provengan de distintas áreas de estudios y sean capaces de adoptar perspectivas interdisciplinares y de trabajar en equipo (Horwitz \& Horwitz, 2007). Estos estudios enfatizan los efectos positivos de la diversidad en la composición de los equipos de trabajo sobre sus resultados, tanto en el ámbito empresarial como en el ámbito científico. Podríamos catalogar el argumento del valor positivo de la diversidad entre los argumentos utilitaristas: como la diversidad del equipo de trabajo mejora los resultados, me conviene incluir mujeres — bajo la condición de que cumplan con los demás criterios meritocráticos que aplico a cualquier otro miembro del equipoporque esto aumenta la probabilidad de que el resultado del trabajo sea de mejor calidad. La falta de diversidad puede también tener efectos negativos sobre la creatividad e imaginación del grupo y su capacidad de anticipar los deseos y expectativas de los usuarios finales de la solución (Wachter-Boettcher, 2017).

En otras palabras, la ausencia -o limitada presencia- de las mujeres en los equipos que se encargan de diseñar y desplegar soluciones de IA es un problema porque puede limitar el conocimiento general del grupo y el sentido crítico, aumentando el riesgo de que los sesgos del grupo mayoritariamente representados no sean detectados y permeen los sistemas desarrollados (Leavy, 2018). Estudios realizados con datos de España muestran una asociación positiva entre la diversidad de género y la innovación de servicios y productos en el sector manufacturero (Fernández Sastre, 2015). Otros estudios demuestran que la diversidad de género en la alta dirección repercute positivamente en el cambio estratégico, lo que en última instancia mejora el rendimiento de las empresas (Triana et al., 2019). Finalmente, hay efectos positivos, pero no lineales, de la diversidad de los grupos de investigación en la producción científica (De Saá-Pérez et al., 2017).

Existen también razones morales provenientes de las teorías institucionales que justifican la mayor inclusión de mujeres en estos equipos de trabajo y de investigación. Algunas teorías argumentan la necesidad de asegurar cierto nivel de representación de distintos grupos sociales en las instituciones públicas y democráticas para que todos los ciudadanos se sientan representados y perciban esas instituciones como legítimas, benignas y dignas de confianza (Degli Esposti et al., 2021). De hecho, dentro del más amplio debate sobre democracia representativa (Urbinati, 2008), el tema de qué individuos y grupos deberían verse representados en los distintos órganos de gobierno e instituciones públicas está fuertemente relacionado con el tema de la confianza en estas mismas instituciones y su legitimidad. Estudios en esta área demuestran que la presencia equilibrada de mujeres en los órganos de decisiones políticas legitima los procesos de toma de decisiones y confiere confianza y aquiescencia institucional (Clayton et al., 2019). La relación entre representación simbólica y de identidad, confianza institucional y legitimidad del proceso de toma de decisiones colectivas se materializa en todo tipo de institución y organización, tanto en entidades públicas como en entidades privadas. En otras palabras, el hecho de que nos reconozcamos y que podamos identificarnos 
con personas que desempeñan determinados cargos aumentará nuestro interés en sus actividades. A partir de este interés valoraremos si fiarnos de ellos y si nos dejaremos influenciar por sus acciones y opiniones.

En resumen, varios estudios demuestran las ventajas de aumentar la diversidad en la composición de los equipos de trabajo al fin de mejorar sus conocimientos, creatividad y sentido crítico. La segunda teoría de la legitimidad y confianza institucional, propone además que la inclusión de mujeres en equipos de $\mathrm{I}+\mathrm{D}+\mathrm{i}$ de IA aumentaría el nivel de representación del colectivo femenino en esos órganos de decisión, aumentando la legitimidad de las decisiones tomadas por ellos. Ese aumento de legitimidad se debería a que los grupos representados considerarían que las personas con las que comparten identidades colectivas actuarían de acuerdo a las normas sociales compartidas en esos grupos y tomarían decisiones que no afectarían negativamente a sus semejantes. Claramente, estas asunciones sobre si estas expertas son de fiar serían sucesivamente desmentidas o confirmadas en base al comportamiento adoptado por parte de ellas y de las organizaciones que representan. En base a estas dos teorías se puede argumentar que la presencia de mujeres especialistas en IA debería mejorar tanto la calidad como la aceptación de estas tecnologías. En este estudio no pondremos a prueba ${ }^{5}$ la validez de estas hipótesis. Nos limitaremos a tomar estas hipótesis como punto de partida y a calcular, en base a un análisis descriptivo, si habría margen para aumentar el porcentaje de mujeres especialistas TIC (Tecnologías de la Información y la Comunicación) en equipos de desarrollo de soluciones de IA y, así, cumplir con las recomendaciones provenientes de este marco teórico.

\footnotetext{
5 Validar estas teorías implicaría analizar datos que relacionen la composición de grupos de desarrolladores de tecnologías de IA con, por ejemplo, la venta de esos productos. Se podría también llevar a cabo un estudio cualitativo en el que se evalúe si la presencia de mujeres ha influido en el desarrollo de productos o servicios de IA, produciendo cambios basados en consideraciones de género.
}

5. El análisis de género y los puntos ciegos de la IA

Es preciso recordar que la incorporación del análisis de género en el diseño experimental ha permitido avances en muchas disciplinas (Tannenbaum et al., 2019). En ingeniería, por ejemplo, considerar a las personas de baja estatura (muchas mujeres, pero también muchos hombres) conductores «fuera de posición» conlleva mayores lesiones en accidentes de tráfico (Carter et al., 2014). Entre 1997 y 2000, se retiraron del mercado estadounidense diez medicamentos por sus efectos nocivos para la salud. Ocho de ellos suponían «mayores riesgos para la salud de las mujeres que de los hombres» (USGAO, 2001).

La consideración de enfoques que analicen la cuestión de género puede añadir dimensiones valiosas a la investigación y así maximizar la diversidad no solo en los equipos de investigación, sino también en los métodos y en las preguntas de investigación (Nielsen et al., 2018). De hecho, la Comisión Europea, en colaboración con la US National Science Foundation y la Universidad de Stanford, promueve las «Innovaciones de género» (en inglés, Gendered Innovations) $)^{6}$, es decir, el uso de perspectivas que analicen el poder creativo del sexo, del género y del análisis interseccional para la innovación y el descubrimiento (European Commission, 2020b). De forma similar, en el art. 33.1. j) de la Ley 14/2011, de 1 de junio, de la Ciencia, la Tecnología y la Innovación («BOE» núm. 131, de 2 de junio de 2011), se hace hincapié en las «medidas para la inclusión de la perspectiva de género como categoría transversal en la ciencia, la tecnología y la innovación, y para impulsar una presencia equilibrada de mujeres y hombres en todos los ámbitos del Sistema Español de Ciencia, Tecnología e Innovación»; además, en al art. 14.1. b) se exige el «respeto al principio de igualdad de género en el

6 http://genderedinnovations.stanford.edu/ 
desempeño de las funciones investigadoras, en la contratación de personal y en el desarrollo de la carrera profesional».

Cuando los prejuicios de género se convierten en sesgos algorítmicos se pueden perder inversiones, oportunidades de mercado, de trabajo, etcétera. El tema de la equidad (en inglés, fairness) de los algoritmos de clasificación se convierte en una nueva área de desarrollo técnico (Dwork et al., 2018), y a su vez en un debate más amplio sobre los individuos, los grupos, las organizaciones y las instituciones responsables de la programación e implementación a gran escala de estos sistemas automatizados de toma de decisiones. En el ámbito de PLN, hoy en día, existen dos estrategias fundamentales para mitigar los sesgos de género a la hora de desarrollar algoritmos de procesamiento del lenguaje natural: la mitigación de sesgos en los corpora lingüísticos de entrenamiento y el ajuste de parámetros y modelos usados (Sun et al., 2019).

Numerosos estudios del lenguaje han identificado rasgos lingüísticos recurrentes que son atribuibles al sesgo de género (Leavy, 2018). Utilizando un método de incrustación de palabras en un corpus de seis mil millones de palabras en inglés, un estudio confirma que el rosa, el azul y el rojo tienen un sesgo hacia la positividad, pero, además, el rosa tiene un claro sesgo de género y se usa para trasmitir femineidad (Jonauskaite et al., 2021). Otro análisis de los adjetivos utilizados para describir hombres y mujeres en los periódicos británicos demuestra que es más frecuente que los hombres sean descritos en términos de su comportamiento, mientras que las mujeres lo sean en términos de su apariencia y sexualidad (Caldas-Coulthard et al., 2010).

La falta de información sobre un determinado grupo puede producir una variación en el grado de fiabilidad del algoritmo que usaremos para clasificar los elementos de dicho conjunto de datos. Un ejemplo muy famoso es el alto nivel de error a la hora de identificar fotos de mujeres negras respecto a la identificación de hombres blancos presente en la misma base de datos usada para entrenar el sistema (Buolamwini \& Gebru,
2018). Este mismo problema, pero con mayores riesgos para las personas, se puede dar en el caso de sistemas que intenten predecir la probabilidad de que una persona que ha sido condenada, vuelva a cometer un delito y basar en esta predicción la decisión de que salga o se quede en la cárcel (Flores et al., 2016; Angwin et al., 2016).

Ejemplos de resultados prejudiciales inesperados debido a sesgos algorítmicos se han dado en áreas de aplicación muy diversas. Además, determinados atributos personales, como, por ejemplo, las variables sociodemográficas clásicas (etnia, género, código postal, etc.), así como la presencia de indicadores de preferencias y comportamientos individuales en línea (likes, retweet, share, searches, etc.) y su procesamiento a gran escala, abren la posibilidad de que patrones culturales, estereotipos y prejuicios que normalmente se manifiestan de forma difuminada en el día a día, se impongan como modelos dominantes a seguir y que eso perjudique el resultados de decisiones importantes para las personas. Consideremos algunos ejemplos.

Un algoritmo programado para optimizar la entrega rentable de anuncios comerciales que pretenden ser neutros desde el punto de vista del género puede terminar ofreciendo anuncios de trabajos de Ciencia, Tecnología, Ingeniería y Matemáticas (STEM, por sus siglas en inglés) más a hombres que a mujeres. Este comportamiento podría ser interpretado como discriminatorio cuando en realidad el desequilibrio se genera porque las mujeres en las subastas de publicidad digital son un grupo demográfico muy apreciado $\mathrm{y}$, por tanto, más caro a la hora de mostrarles anuncios (Lambrecht \& Tucker, 2019). La única forma de detectar y corregir estos sesgos es a través del análisis de sistemas sociotécnicos realizada por equipos interdisciplinares.

\section{Diversidad e inclusión para contrarrestar los sesgos algorítmicos}

Aunque por diversidad de género se entiende comúnmente la composición de género de los equipos 


\section{FIGURA 1}

EVOLUCIÓN DEL ÍNDICE DE ECONOMÍA Y SOCIEDAD DIGITAL (DESI) EN ESPAÑA, PORTUGAL, IRLANDA Y LA MEDIA DE LOS 28 PAISES DE LA UE ENTRE 2015 Y 2020

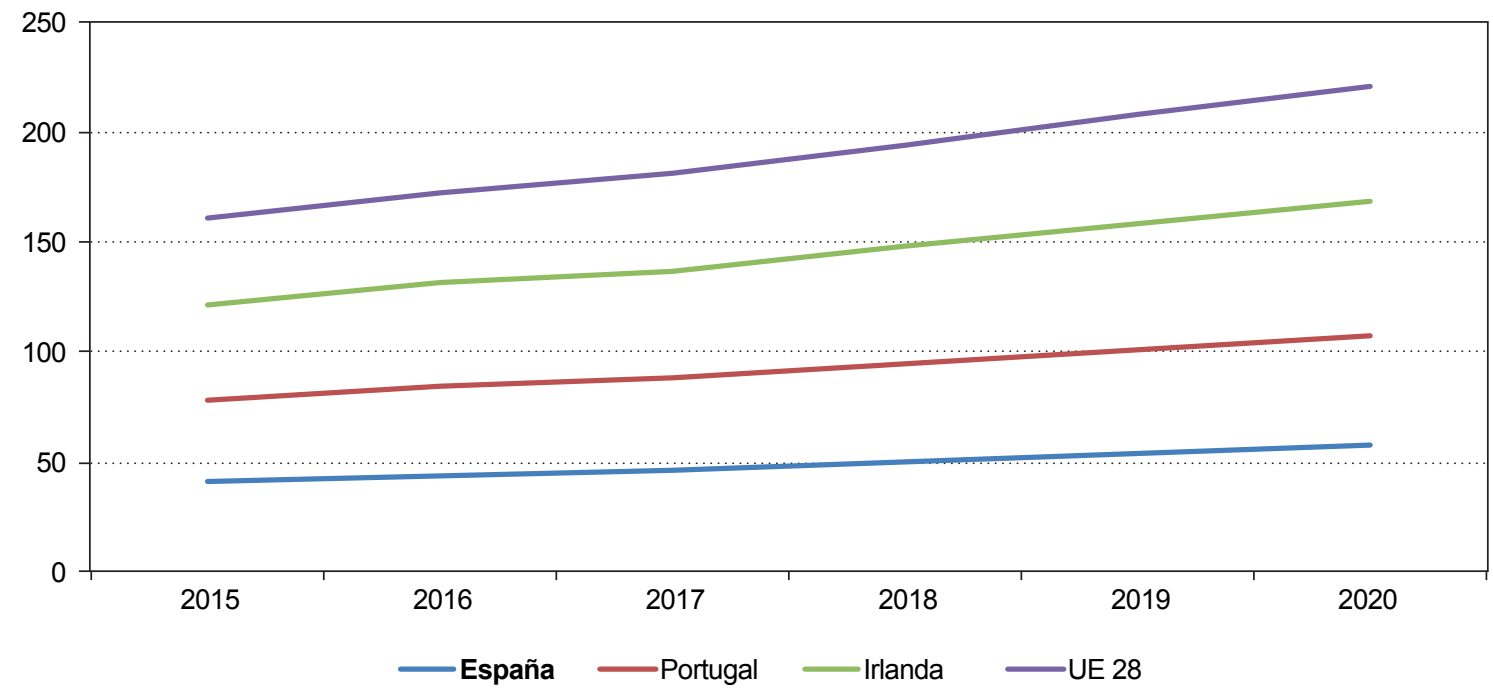

NOTA: https://digital-strategy.ec.europa.eu/en/policies/desi

FUENTE: Elaboración propia de datos del Digital Economy and Society Index (DESI).

de trabajo o de investigación, la plena realización del potencial de la diversidad para la ciencia y la innovación requiere también que se preste atención a los métodos empleados y a las cuestiones que se plantean en la elaboración del conocimiento técnico y científico. Por supuesto podemos notar cierta recursividad y endogeneidad en estos elementos. Por un lado, necesitaremos mujeres que hayan estudiado carreras técnicas relacionadas con el big data y la IA. Podríamos también, por otro lado, incluir mujeres que provengan de otras áreas de estudios relacionadas con el análisis de la aceptación de la tecnología, la percepción del riesgo tecnológico, la relación humano-máquina, el derecho TIC, la economía de las plataformas digitales, etc. La inclusión de expertas con estos perfiles cumpliría con lo previsto por nuestro marco teórico: la presencia de tecnólogas aumentaría el nivel de representación de ese colectivo y la presencia de expertas de áreas de estudios complementarias a las áreas técnicas fomentaría la multidisciplinariedad en la investigación en IA. Se aumentaría la diversidad de la composición del equipo, lo que aumentaría su potencial creativo e imaginativo.

El primer obstáculo que nos impide cumplir con las recomendaciones que provienen del marco teórico aquí presentado es ampliamente conocido y se refiere a la falta de mujeres estudiando carreras de informáticas o de otras áreas de estudio relacionadas. Faltan tanto graduadas como especialistas TIC. El Índice de Economía y Sociedad Digital (DESI, por sus siglas en inglés) publicado en junio de 2020 por la Comisión Europea combinando valores de hasta 37 indicadores, asigna a España una puntuación de 13,3 sobre 100 en la categoría «Graduados TIC», 


\section{FIGURA 2}

\section{DISTRIBUCIÓN DEL ALUMNADO POR CAMPO DE ESTUDIOS EN EL CURSO 2018-2019} (En \%)

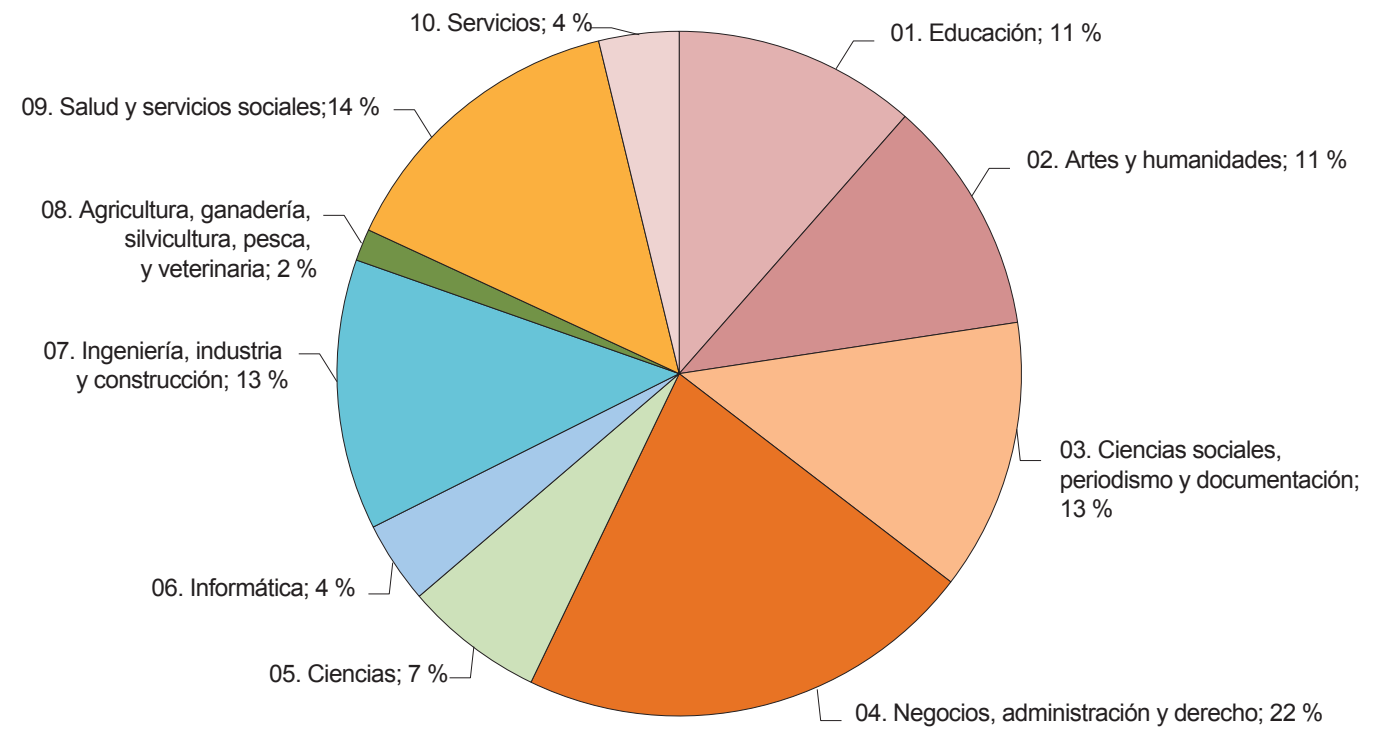

FUENTE: Elaboración propia de datos del SIIU (2020a).

15,2 en la categoría «Personas empleadas con habilidades de especialista en TIC» y 9,49 en la categoría «Mujeres empleadas con habilidades de especialista en TIC». Estos dos últimos indicadores son inferiores a la puntuación obtenida por la media de los 28 países de la UE. A pesar de ocupar la segunda posición en la dimensión de Servicios Públicos Digitales por detrás de Estonia hay margen de mejora en comparación con otros Estados miembros (véase Figura 1) en términos de competitividad en lo relativo a la Economía y Sociedad Digital.

\section{En búsqueda de futuras especialistas en IA}

Asumiendo que las más capacitadas para realizar un análisis de género sean las expertas de IA, la primera pregunta es qué tipo de expertas necesitamos y dónde podemos encontrarlas. Según datos del «Observatorio Mujeres, Ciencia e Innovación» (OMCl) ${ }^{7}$, las mujeres representan el $56 \%$ de los 1.309 .791 estudiantes matriculados en estudios de grado de primer y segundo ciclo en el curso 2019-2020. Este dato mejora las cifras del curso 2018-2019, en el que las mujeres eran el $55 \%$ del alumnado total (1.290.346) en el «Sistema Universitario Español» (SUE) conformado por 83 universidades en total, de las cuales 50 son públicas y 33 privadas. Mientras que la distribución de estudiantes por campo de estudio es relativamente equilibrada (véase la Figura 2), se nota cierta preferencia por las áreas de «Humanidades y Ciencias Sociales» $(57 \%)$ respecto a

\footnotetext{
${ }^{7} \mathrm{OMCl}, \mathrm{CIENTÍFICAS} \mathrm{EN} \mathrm{CIFRAS} \mathrm{2021,} \mathrm{https://www.lamoncloa.}$ gob.es/serviciosdeprensa/notasprensa/ciencia-e-innovacion/ Documents/2021/080321-\%20Cientificas_en_Cifras_2021.pdf
} 
TABLA 1

\section{NÚMERO DE ESTUDIANTES MATRICULADOS EN GRADO EN EL CURSO 2019-2020 EN TODAS LAS ÁREAS DE ESTUDIO POR GÉNERO}

\begin{tabular}{|c|c|c|c|}
\hline & \multicolumn{3}{|c|}{ CURSO 2019-2020 } \\
\hline & $\%$ Hombres & $\%$ Mujeres & Total \\
\hline 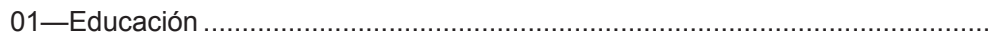 & 22 & 78 & 148.069 \\
\hline 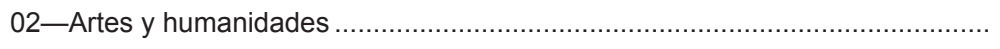 & 39 & 61 & 143.579 \\
\hline 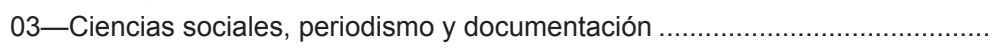 & 38 & 62 & 165.264 \\
\hline 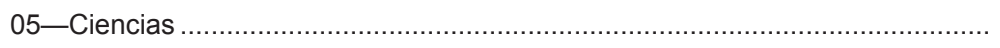 & 51 & 49 & 85.226 \\
\hline 06-Informática & 87 & 13 & 49.979 \\
\hline 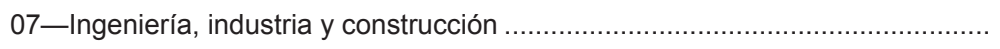 & 71 & 29 & 164.903 \\
\hline 08 -Agricultura, ganadería, silvicultura, pesca y veterinaria............................. & 47 & 53 & 19.462 \\
\hline 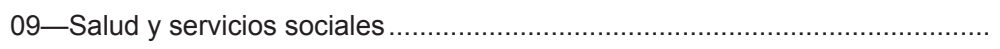 & 29 & 71 & 184.808 \\
\hline 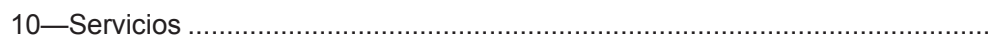 & 58 & 42 & 48.829 \\
\hline
\end{tabular}

las áreas de «Ciencias» e «Ingeniería» (23\%), seguidas por «Salud y servicios sociales» (14\%), «Servicios» (4\%) y «Agricultura, ganadería, silvicultura, pesca y veterinaria» $(2 \%)$.

Se muestra en la Tabla 1 que el porcentaje de hombres matriculados en informática ${ }^{8}$ en el curso 2019 2020 es del $87 \%$; hay solamente 7.086 alumnas de informáticas, es decir un $13 \%$ de los 52.892 estudiantes matriculados (SIIU, 2020a). Estos datos reflejan la tendencia general en otros países desarrollados. Según la encuesta Taulbee de la Computing Research Association sobre estudiantes de ingeniería informática en EE UU y Canadá, en el curso 2016-2017 solo el 19,2 \% de los títulos de grado, el $29,6 \%$ de los másteres y el $19,3 \%$ de los títulos de doctorado se otorgaron a mujeres. Aunque este último dato mejore en $2019-2020$ —el $21,7 \%$ de los

\footnotetext{
${ }_{8}$ Se incluye en este grupo los estudiantes matriculados en grados de: Informática, Ingeniería multimedia, Desarrollo de videojuegos, Desarrollo de software y de aplicaciones, Diseño y administración de base de datos y redes.
}

doctores en computación son mujeres- hay un evidente desequilibrio de género en EE UU (Zweben \& Bizot, 2020).

La marginalidad de las mujeres en las áreas de estudio de informática es llamativa incluso en comparación con otras áreas de estudios complementarias. Por ejemplo, las alumnas de «Matemáticas» representan el $37 \%$ del total de 11.918 estudiantes en 20182019. Sin embargo, hay que evidenciar una tendencia positiva en el tiempo. En el curso 2018-2019 el número total de alumnas de Informática son 6.447 , un considerable aumento respecto al curso 2017-2018 cuando las estudiantes de informática eran solamente $2.115^{9}$. Además, si nos centramos en el curso 2018-2019, podemos añadir a las 6.447 alumnas de «Informática» las alumnas de otras áreas de estudios complementarias, como «Ingeniería de Telecomunicación»

\footnotetext{
9 EDUCAbase, Estudiantes matriculados por tipo y modalidad de la universidad, sexo, dedicación al estudio y campo de estudio (solo Grado), Ministerio de Educación y Formación Profesional / Ministerio de Universidades.
} 
TABLA 2

\section{NÚMERO DE ESTUDIANTES MATRICULADOS EN GRADO EN EL CURSO 2018-2019 EN TODAS LAS ÁREAS DE ESTUDIO POR GÉNERO}

\begin{tabular}{|c|c|c|c|}
\hline \multirow[b]{2}{*}{ Campo de estudio } & \multicolumn{3}{|c|}{ Estudiantes matriculados en Grado } \\
\hline & $\%$ Hombres & $\%$ Mujeres & Total \\
\hline 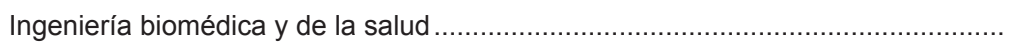 & 39 & 61 & 2.458 \\
\hline 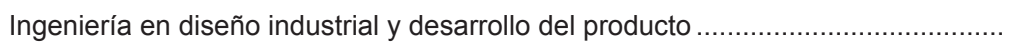 & 53 & 47 & 7.123 \\
\hline 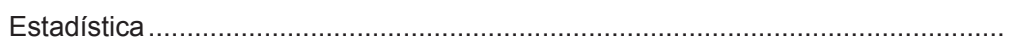 & 55 & 45 & 1.742 \\
\hline Otras matemáticas y estadística ........................ & 70 & 30 & 222 \\
\hline 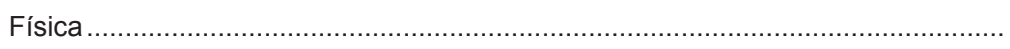 & 73 & 27 & 10.828 \\
\hline 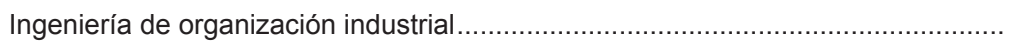 & 73 & 27 & 6.270 \\
\hline 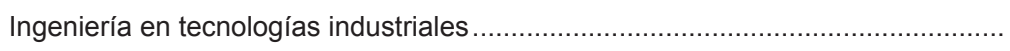 & 76 & 24 & 16.941 \\
\hline 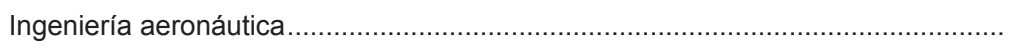 & 76 & 24 & 7.108 \\
\hline 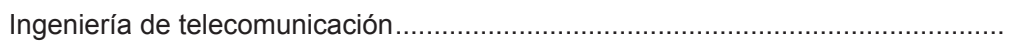 & 79 & 21 & 12.591 \\
\hline Ingeniería naval y oceánica.............................. & 80 & 20 & 2.709 \\
\hline 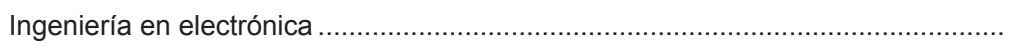 & 83 & 17 & 4.509 \\
\hline 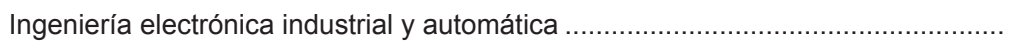 & 85 & 15 & 15.079 \\
\hline 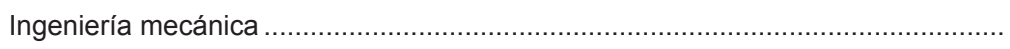 & 87 & 13 & 22.920 \\
\hline Informática......................................... & 87 & 13 & 49.979 \\
\hline 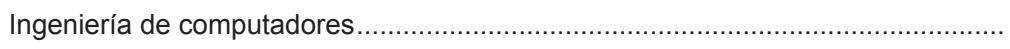 & 90 & 10 & 2.098 \\
\hline 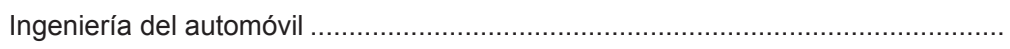 & 94 & 6 & 377 \\
\hline
\end{tabular}

(2.634), «Matemáticas» (4.404), «Estadística» (791), o «Física» (2.893).

En la Tabla 2 podemos ver el listado completo de grados potencialmente relacionados con el desarrollo de la IA. Si miramos al porcentaje de mujeres en cada caso, veremos que hay variaciones en términos de paridad entre hombres y mujeres, con una clara inversión de tendencia en el área de «Ingeniería biomédica y de la salud», en la que las mujeres representan el $61 \%$ de alumnado. "Ciencias», de hecho, llega a ser un área paritaria gracias a grados como «Biología» en el que el $62 \%$ de los 17.196 estudiantes matriculados son mujeres.

Para poder terminar esta exploración de la presencia femenina en las áreas de estudios afines a la investigación en IA y big data, procede calcular el número de estudiantes matriculados en cursos de Formación Profesional (FP). En el curso 20192020 las mujeres matriculadas en «Informática y Comunicaciones» en la FP básica son 2.200, en el grado medio suman 2.360 , en el grado superior alcanzan 4.300 en modalidad presencial y 2.706 en modalidad a distancia (EDUCAbase, 2021a, 2021b, 2021c, 2021d).

A estas estudiantes podemos añadir las 1.430 de «Electricidad y Electrónica», las 756 de «Animaciones 3D, juegos y entornos interactivos», y las 427 de «Sistemas microinformáticos y redes». En total las mujeres matriculadas en los varios ciclos de FP de interés alcanzan 14.179 alumnas. Esta cifra más las 36.374 alumnas de 
TABLA 3

NÚMERO DE ESTUDIANTES MATRICULADOS EN CICLOS FORMATIVOS DE FORMACIÓN PROFESIONAL BÁSICA, DE GRADO MEDIO Y SUPERIOR EN MODALIDAD PRESENCIAL O A DISTANCIA EN ÁREAS RELACIONADAS CON LA IA EL CURSO 2018-2019 POR GÉNERO

\begin{tabular}{|c|c|c|c|c|}
\hline Tipo de FP & Ciclo formativo & $\begin{array}{c}\% \\
\text { Hombres }\end{array}$ & $\begin{array}{c}\% \\
\text { Mujeres }\end{array}$ & Total \\
\hline FP básica & Informática y Comunicaciones & 83 & 17 & 12.672 \\
\hline Grado medio presencial & Informática y Comunicaciones & 93 & 7 & 33.559 \\
\hline Ciclos formativos de grado superior presencial & Electricidad y Electrónica* & 94 & 6 & 23.396 \\
\hline Ciclos formativos de grado superior presencial & Animaciones 3D, juegos y entornos interactivos & 81 & 19 & 3.998 \\
\hline Ciclos formativos de grado superior presencial & Informática y Comunicaciones** & 90 & 10 & 42.237 \\
\hline Ciclos formativos de grado medio a distancia & Sistemas microinformáticos y redes & 85 & 15 & 2.809 \\
\hline Ciclos formativos de grado medio a distancia & Electricidad y Electrónica* & 91 & 9 & 750 \\
\hline Ciclos formativos de grado medio a distancia & Informática y Comunicaciones** & 81 & 19 & 14.319 \\
\hline \multicolumn{5}{|c|}{$\begin{array}{l}\text { NOTAS: * Incluye: Automatización y robótica industrial; Electromedicina clínica; Mantenimiento electrónico; Sistemas de telecomunicaciones } \\
\text { e informáticos; Sistemas electrotécnicos y automatizados. ** Incluye: Administración de sistemas informáticos en red; Desarrollo de } \\
\text { aplicaciones multiplataforma; Desarrollo de aplicaciones web. } \\
\text { FUENTE: Elaboración propia de datos del Ministerio de Educación y Formación Profesional (EDUCAbase, 2021a, 2021b, 2021c, } \\
\text { 2021d). }\end{array}$} \\
\hline
\end{tabular}

grado compone un total de 50.553 estudiantes. A estas habría que añadir las estudiantes de másteres oficiales y títulos propios en áreas como big data y ciencias de datos y las estudiantes de doctorado en las escuelas politécnicas superiores y en las facultades de ingeniería informática y de telecomunicaciones.

\section{Hacia la interdisciplinariedad en la I+D+i de IA}

El segundo obstáculo es menos conocido y deriva de la rigidez del sistema académico español y de la falta de mecanismos y organismos que fomenten y premien la investigación interdisciplinaria. Analizaremos brevemente en esta segunda parte cada uno de estos problemas. En 2017 era solo un $12 \%$ el porcentaje de mujeres autoras de trabajos aceptados en tres de las principales conferencias de aprendizaje automático (Simonite, 2018). Solo el $18 \%$ de los autores de las principales conferencias sobre IA son mujeres, y más del $80 \%$ de los profesores de IA son hombres (Gagne, 2019).
En el corto plazo es imposible cambiar radicalmente estas cifras. Esta disparidad se refleja de forma extrema en la industria de la IA: las mujeres solo representan el $15 \%$ del personal de investigación en IA en Facebook y el $10 \%$ en Google (Simonite, 2018). Solo el $22 \%$ de los profesionales mundiales de la IA son mujeres y solo un $2 \%$ de empresas tecnológicas españolas están dirigidas por ellas. El «Mapa de capacidades de tecnologías de $\mid A »^{10}$ elaborado por el Gobierno a través del «Grupo Interministerial en Inteligencia Artificial» creado bajo la «Comisión Delegada para Política Científica, Tecnológica y de Innovación» nos muestra que las empresas que usan o desarrollan IA son pymes o tienen equipos de I+D+i de IA bastante pequeños: el $62 \%$ tienen menos de 11 empleados dedicados a tareas de IA.

La segunda propuesta para solucionar el problema del desequilibrio de género en la producción de la IA es

\footnotetext{
10 https://mapa.estrategiaia.es/
} 


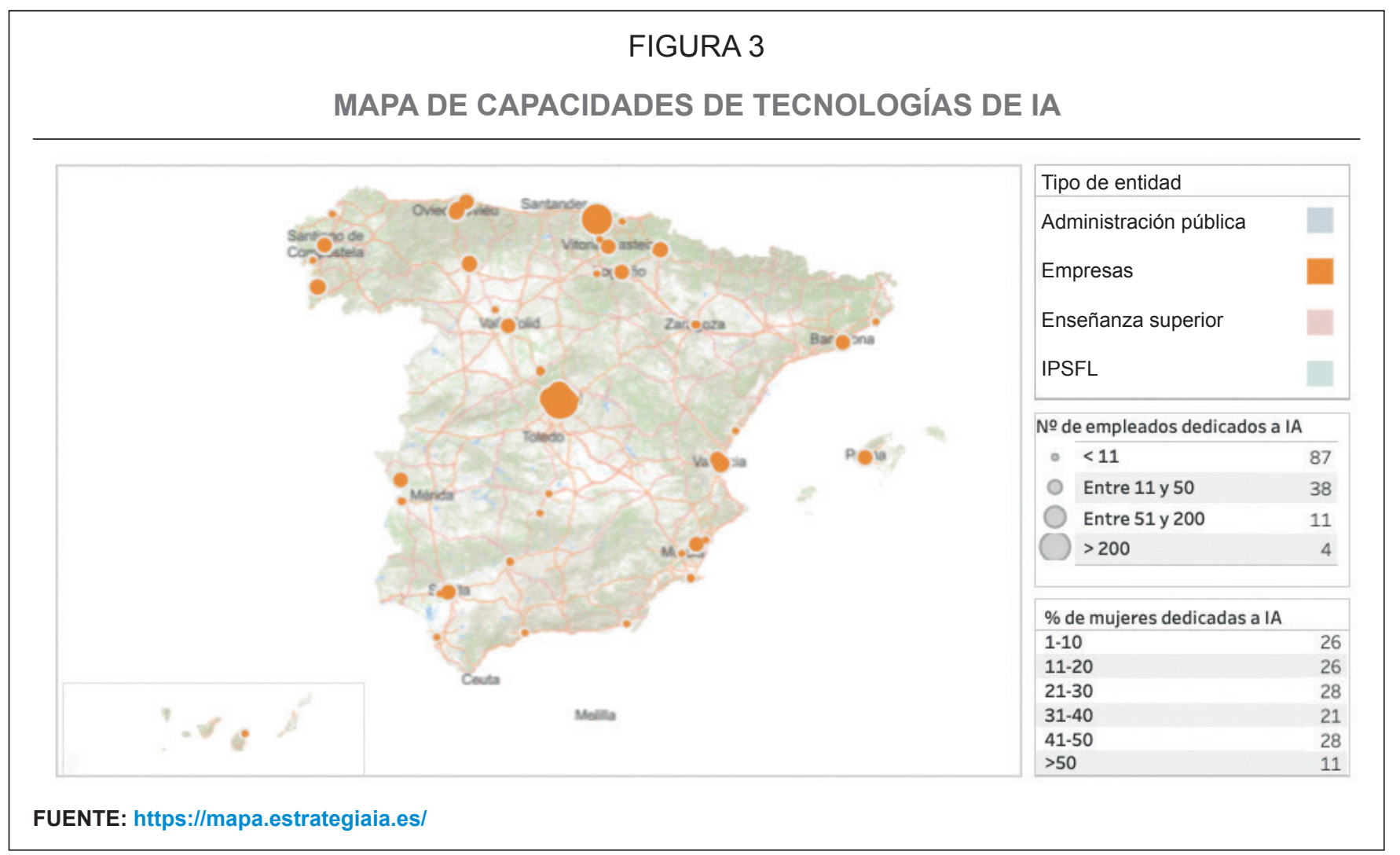

a través de la construcción de equipos interdisciplinarios de trabajo a los que se incorporen analistas procedentes de disciplinas de artes, humanidades y ciencias sociales y jurídicas, que puedan abarcar los grandes retos jurídicos y sociales que acompañan la transformación digital y la vinculación de la cantidad ingente de datos a procesar y analizar con todos los ámbitos sociales y económicos como la salud, la educación o la política (Sáinz et al., 2020).

Varios obstáculos hoy en día impiden establecer estas colaboraciones entre expertas y expertos de distintas áreas de estudio que trabajan en organismos públicos y privados. El proceso de acreditación de la ANECA (Agencia Nacional de Evaluación de la Calidad y Acreditación) genera desincentivos a publicar en revistas fuera del área de estudio del personal docente e investigador. Es difícil encontrar revistas reconocidas en áreas tan distantes como ciencias sociales $\mathrm{y}$ computación. Derecho representa un nicho epistémico con reglas de acreditación muy estrictas que no reflejan la cambiante realidad del derecho TIC, tan dependiente de la colaboración con tecnólogos. Ámbitos de estudio transversales como la ciberseguridad y la protección de datos -áreas de reconocida utilidad y altamente demandadas por el mundo empresarial— no se ven representados en los departamentos de muchas universidades. La diferencia de condiciones laborales ofrecida por las empresas y por los organismos públicos de investigación deja a menudo a las universidades sin docentes e investigadores expertos en IA.

A la ausencia de mujeres se añade la ausencia de profesorado y la necesidad de establecer colaboraciones con empresas para volver a tener acceso al conocimiento de los expertos. Otro problema relacionado es que la mayoría de la inversión en I+D+i en IA viene del sector privado y de la investigación llevada a cabo por 
empresas específicas. En este sentido, es útil mencionar el Real Decreto 1529/2012 ${ }^{11}$, de 8 de noviembre, y la Orden ESS/2518/2013 12 que establecen las bases de la «Formación Profesional Dual», una nueva modalidad dentro de la Formación Profesional. Estos proyectos se caracterizan por realizarse en régimen de alternancia entre el centro educativo y la empresa, con un número de horas o días de estancia de duración variable entre el centro de trabajo y el centro educativo. A pesar de que la Formación Dual pretende incrementar la vinculación y corresponsabilidad del tejido empresarial con la Formación Profesional y, así, favorecer la transferencia de conocimientos entre centros educativos y empresas, la utilidad de la implementación de este programa está relacionada con el nivel de inversión en I+D+i en IA de estas entidades y con la real capacidad de poder dedicar parte de la jornada laboral del personal de plantilla a actividades de formación. Hay también que notar que la gran mayoría de las empresas no desarrolla productos de IA, sino que son usuarios de soluciones vendidas por multinacionales con sede en otros países. Aunque el mercado laboral requiera certificaciones de la capacidad de usar esos productos, su conocimiento poco aporta a que estos trabajadores tengan la capacidad de desarrollar nuevos productos. La innovación en IA surge normalmente de la creación de start-ups por parte de estudiantes de grado y posgrado en contexto que fomenten el espíritu emprendedor y donde haya disponibilidad de capital de riesgo. La creación de parques tecnológicos que unan universidades y empresas, facilitando la creación de start-ups, podría ayudar a generar el adecuado entorno que permita el trasvase de

\footnotetext{
${ }_{11}$ Real Decreto 1529/2012, de 8 de noviembre, por el que se desarrolla el contrato para la formación y el aprendizaje y se establecen las bases de la formación profesional dual. «BOE» núm. 270, de 9 de noviembre de 2012, páginas 78348 a 78365 .

${ }_{12}$ Orden ESS/2518/2013, de 26 de diciembre, por la que se regulan los aspectos formativos del contrato para la formación y el aprendizaje, en desarrollo del Real Decreto 1529/2012, de 8 de noviembre, por el que se desarrolla el contrato para la formación y el aprendizaje y se establecen las bases de la formación profesional dual. «BOE» núm. 10, de 11 de enero de 2014, páginas 1560 a 1568 .
}

conocimiento entre generaciones próximas como los estudiantes de secundaria y los estudiantes de grado y posgrado. Las barreras de género no son las únicas barreras a la innovación de hecho. La constante evolución de los lenguajes de programación y de los instrumentos de cómputo requiere reducir las distancias generacionales entre los que consideramos alumnos y profesores.

\section{Conclusión}

Hoy en día existe una problemática derivada de la limitada presencia de mujeres en los ámbitos asociados con el diseño y desarrollo de la inteligencia artificial (IA) y de las tecnologías de macrodatos (big data). En este artículo nos hemos centrado en dos argumentos fundamentales a favor de la inclusión de mujeres y del análisis de género en tareas relacionadas con el diseño y desarrollo de estas tecnologías. Por un lado, hemos hablado del beneficio en términos de creatividad y capacidad innovadora de una mayor diversidad en la composición de los equipos de trabajo y de investigación en IA y big data. Por otro lado, hemos hablado de la necesidad de que las mujeres, en calidad de importante colectivo social, se vean representadas en el diseño de tecnologías digitales que tienen un gran impacto sobre la sociedad. Además, hemos destacado la importancia de fomentar la inter y multidisciplinariedad facilitando la colaboración entre expertas de distintas áreas de conocimientos, desde la ingeniería hasta las ciencias sociales y las humanidades. Creyendo en la validez del marco teórico anteriormente expuesto, el análisis descriptivo realizado en el estudio ha permitido identificar áreas de estudio propicias para formar especialistas TIC y expertas capaces de desarrollar nuevas expresiones de análisis de género que nos ayuden a combatir los sesgos algorítmicos derivados de la falta de inclusión y visibilidad de la mujer, tanto en los macrodatos como en la I+D+i de IA.

En base a la estimación del número de mujeres matriculadas en las áreas de estudios y ciclos 
formativos relacionados con la informática, la ingeniería y la ciencia de datos, podemos llegar a afirmar que España cuenta con aproximadamente 50.000 nuevas potenciales especialistas TIC cada año sobre un total de trabajadores activos ocupados de 19.206 .800 , es decir un $0,26 \%$ de potenciales nuevas trabajadoras que podrían unirse a la Seguridad Social en sectores relacionados con servicios y tecnologías digitales de alto valor añadido y que cada vez más favorecen el teletrabajo y la flexibilidad del horario laboral. No sabemos el tipo de tecnologías que estas especialistas TIC imaginarían. Sin embargo, si tomamos como ejemplo la labor de Carme Torras, Premio Nacional de Investigación 2020, investigadora en Instituto de Robótica e Informática Industrial (IRI) del CSIC, veremos cómo su grupo de investigación ha decidido centrarse en desarrollar sistemas robóticos interactivos de asistencia. Sistemas que se enfrentan a lo cotidiano y abarcan tareas como doblar prendas o ayudar a personas enfermas o ancianas a vestirse. Tareas fáciles de realizar por humanos y que sin embargo siguen desafiando las máquinas y su «inteligencia».

La innovación puede surgir de una invención, es decir de una forma totalmente nueva de hacer las cosas, o de la imitación, es decir, de una réplica con variación de nuestra forma de hacer. La introducción de la perspectiva de género en el diseño y desarrollo de tecnologías digitales como el big data o la IA puede ayudar a desmitificar el carácter disruptivo y de cambio ex abrupto de la innovación y ayudarnos a ver la continuidad, progresión y repeticiones típicas de los sistemas sociotécnicos. Además, nos puede ayudar a ver y prevenir los prejuicios y sesgos que se albergan tanto en las máquinas como en los humanos. La reproducción de los sesgos de género, tanto en la sociedad como en los algoritmos, es una expresión de la profunda continuidad que existe en los hechos históricos, sociales y tecnológicos. Una innovación verdaderamente novedosa debería ser capaz de romper antiguas cadenas dibujando nuevas formas de prosperar y coexistir.
Probablemente no tendremos mucho más de 50.000 mujeres especialistas TIC durante varios años en España. Sin embargo, si empezáramos a conocer mejor su trabajo y su experiencia, seríamos capaces de visibilizar, evaluar y valorar sus aportaciones y convertir estas contribuciones en nuevos modelos de innovación a seguir.

\section{Referencias bibliográficas}

Angwin, J., Larson, J., Mattu, S., \& Kirchner, L. (2016). Machine Bias. There's software used across the county to predict future criminals. And it's biased against blacks. ProPublica, 23, 77-91. https://www. propublica.org/article/ machine-bias-risk-assessments-in-criminal-sentencing

Buolamwini, J., \& Gebru, T. (2018). Gender shades: Intersectional accuracy disparities in commercial gender classification. In S. A. Friedler, \& C. Wilson (eds.), Conference on Fairness, Accountability and Transparency, 81 (pp, 77-91). PMLR.

Caldas-Coulthard, C. R., \& Moon, R. (2010). 'Curvy, hunky, kinky': Using corpora as tools for critical analysis. Discourse \& Society, 21(2), 99-133.

Carter, P. M., Flannagan, C. A., Reed, M. P., Cunningham, R. M., \& Rupp, J. D. (2014). Comparing the effects of age, $\mathrm{BMI}$ and gender on severe injury (AIS 3+) in motor-vehicle crashes. Accident Analysis \& Prevention, 72, 146-160.

Clayton, A., O’Brien, D. Z., \& Piscopo, J. M. (2019). All male panels? Representation and democratic legitimacy. American Journal of Political Science, 63(1), 113-129.

De Saá-Pérez, P., Díaz-Díaz, N. L., Aguiar-Díaz, I., \& Ballesteros-Rodríguez, J. L. (2017). How diversity contributes to academic research teams performance. R\&d Management, 47(2), 165-179.

Degli Esposti, S., Ball, K., \& Dibb, S. (2021). What's In It For Us? Benevolence, National Security, and Digital Surveillance. Public Administration Review, 1-12. https:// doi.org/10.1111/puar.13362

Del Giudice, M. (2017). Pink, blue, and gender: An update. Archives of sexual behavior, 46(6), 1555-1563.

Dencik, L., Hintz, A., Redden, J., \& Treré, E. (2019). Exploring Data Justice: Conceptions, Applications and Directions. Information, Communication \& Society, 22(7), 873-881. https://doi.org/10.1080/1369118X.2019.1606268

Dwork, C., Immorlica, N., Kalai, A. T., \& Leiserson, M. (2018). Decoupled Classifiers for Group-Fair and Efficient Machine Learning. In Proceedings of the 1st Conference on Fairness, Accountability and Transparency (pp. 119-133). 
EDUCAbase. (2021a). 1.2 Alumnado matriculado en Ciclos formativos de FP básica por ciclo formativo, titularidad y sexo. Ministerio de Educación y Formación Profesional, Ministerio de Universidades. http://estadisticas.mecd. gob.es/EducaJaxiPx/Tabla.htm?path=/no-universitaria/ alumnado/matriculado/2019-2020-rd/general_ciclosfp// I0/\&file=ciclosfp12.px\&type=pcaxis\&L=0

EDUCAbase. (2021b). 2.3 Alumnado matriculado en Ciclos formativos de grado medio presencial (1) por ciclo formativo, titularidad y sexo. Ministerio de Educación y Formación Profesional, Ministerio de Universidades. http:// estadisticas.mecd.gob.es/EducaJaxiPx/Tabla.htm?path=/ no-universitaria/alumnado/matriculado/2019-2020-rd/ general_ciclosfp//I0/\&file=ciclosfp23.px\&type=pcaxis\&L=0

EDUCAbase. (2021c). 3.2 Alumnado matriculado en Ciclos formativos de grado medio a distancia por ciclo formativo, titularidad y sexo. Ministerio de Educación y Formación Profesional, Ministerio de Universidades. http://estadisticas. mecd.gob.es/EducaJaxiPx/Tabla.htm?path=/nouniversitaria/alumnado/matriculado/2019-2020-rd/general_ ciclosfp//10/\&file=distancia32.px\&type=pcaxis \& $\mathrm{L}=0$

EDUCAbase. (2021d). 5.2 Alumnado matriculado en Ciclos formativos de grado superior a distancia 1) por ciclo formativo, titularidad y sexo. Ministerio de Educación y Formación Profesional, Ministerio de Universidades. http://estadisticas.mecd.gob.es/EducaJaxiPx/Tabla. htm?path=/no-universitaria/alumnado/matriculado/20192020-rd/general_ciclosfp//I0/\&file=distancia52. px\&type $=$ pcaxis \& $\mathrm{L}=0$

EIGE, European Institute for Gender Equality. (2020). Gender Equality Index. European Institute for Gender Equality. https://eige.europa.eu/gender-equality-index/2020/ES

European Commission. (2018). Communication From The Commission To The European Parliament, The European Council, The Council, The European Economic And Social Committee And The Committee Of The Regions. Coordinated Plan on Artificial Intelligence. COM(2018) 795 final.

European Commission. (2020a). Digital Economy and Society Index (DESI). https://digital-agenda-data.eu/datasets/desi/ visualizations

European Commission. (2020b). Gendered Innovations 2: How inclusive analysis contributes to research and innovation. http://genderedinnovations.stanford.edu/GI\%202\%20 How\%20Inclusive\%20Analysis \%20Contributes $\% 20$ to $\% 20$ R\&l.pdf

Fernández Sastre, J. (2015). The impact of R\&D teams' gender diversity on innovation outputs. International Journal of Entrepreneurship and Small Business, 24(1), 142-162.

Flores, A. W., Bechtel, K., \& Lowenkamp, C. T. (2016). False Positives, False Negatives, and False Analyses: A Rejoinder to Machine Bias: There's Software Used across the Country to Predict Future Criminals. And It's Biased against Blacks. Federal Probation, 80(2), 38.

Fundación COTEC. (2021). Ejecución presupuestaria de la I+D pública (2019). Informe COTEC. https://cotec.es/ observacion/ejecucion-presupuestaria-de-la-i-d-publica/ d70ea91f-2d30-cb54-67d7-bd13e676ee28

Gagne, J. F. (2019). Global Al Talent Report 2019. https:// jfgagne.ai/talent-2019/

Horwitz, S. K., \& Horwitz, I. B. (2007). The effects of team diversity on team outcomes: A meta-analytic review of team demography. Journal of management, 33(6), 987-1015.

Jasanoff, S. (2004). States of knowledge: the co-production of science and the social order. Routledge.

Jonauskaite, D., Sutton, A., Cristianini, N., \& Mohr, C. (2021). English colour terms carry gender and valence biases: $A$ corpus study using word embeddings. PLOS ONE, 16(6), e0251559.

Lambrecht, A., \& Tucker, C. (2019). Algorithmic bias? an empirical study of apparent gender-based discrimination in the display of stem career ads. Management Science, 65(7), 2966-2981.

Leavy, S. (2018). Gender bias in artificial intelligence: The need for diversity and gender theory in machine learning. In Proceedings of the 1st international workshop on gender equality in software engineering (pp. 14-16).

Nielsen, M. W., Bloch, C. W., \& Schiebinger, L. (2018). Making gender diversity work for scientific discovery and innovation. Nature Human Behaviour, 2(10), 726-734.

O'Neil, C. (2017). Weapons of math destruction: How big data increases inequality and threatens democracy. Broadway Books.

Paoletti, J. B. (2012). Pink and blue: Telling the boys from the girls in America. Indiana University Press.

Sáinz, M., Arroyo, L., \& Castaño, C. (2020). Mujeres y digitalización. De las brechas a los algoritmos. Instituto de la Mujer y para la Igualdad de Oportunidades, Ministerio de Igualdad. https://www.inmujeres.gob.es/diseno/ novedades/M_MUJERES_Y_DIGITALIZACION_DE_LAS_ BRECHAS_A_LOS_ALGORITMOS_04.pdf

Serrano, J., Vizcaíno Delgado, D., \& Kalinichenko, B. (2021). Transición digital en la industria europea. Información Comercial Española (ICE), Revista de Economía, 919, 105117. https://doi.org/10.32796/ice.2021.919.7175

SIIU, Sistema Integrado de Información Universitaria. (2020a). Estadística de Estudiantes Universitarios (EEU) Curso 2019-20* (Avance). Sistema Integrado de Información Universitaria (SIIU) y Ministerio de Universidades. Accessed 03/06/2021. https://www.universidades.gob.es/stfls/ MICINN/Universidades/Ficheros/Estadisticas/Principales_ resultados_EEU_19-20.pdf 
SIIU, Sistema Integrado de Información Universitaria. (2020b). Matriculados por tipo y modalidad de la universidad, sexo, dedicación al estudio y campo de estudio (solo Grado), curso 2018-2019. Sistema Integrado de Información Universitaria (SIIU), Secretaría General de Universidades. Accessed 03/06/2021. http://estadisticas.mecd.gob.es/ EducaJaxiPx/Tabla.htm?path=/Universitaria/Alumnado/ Nueva_Estructura/GradoCiclo/Matriculados//I0/\&file=Mat Grad_Sex_Ded_Campo_Tot.px\&type=pcaxis\&L=0

Simonite, T. (2018). Al Is the Future-But Where Are the Women? WIRED, 08/17/2018.

Srnicek, N. (2017). Platform capitalism. John Wiley \& Sons.

Sun, T., Gaut, A., Tang, S., Huang, Y., ElSherief, M., Zhao, J., Mirza, D., Belding, E., Chang, K. W., \& Wang, W. Y. (2019). Mitigating gender bias in natural language processing: Literature review. arXiv preprint arXiv:1906.08976.

Tannenbaum, C., Ellis, R. P., Eyssel, F., Zou, J., \& Schiebinger, L. (2019). Sex and gender analysis improves science and engineering. Nature, 575(7781), 137-146. https://doi. org/10.1038/s41586-019-1657-6

Triana, M. C., Richard, O. C., \& Su, W. (2019). Gender diversity in senior management, strategic change, and firm performance: Examining the mediating nature of strategic change in high tech firms. Research Policy, 48(7), 16811693.

Urbinati, N. (2008). Representative democracy. University of Chicago Press.
USGAO, United States General Accounting Office. (2001). Drug Safety: Most Drugs withdrawn in Recent Years had Greater Health Risks for Women. United States General Accounting Office (Washington, DC: Government Publishing Office). https://www.gao.gov/products/gao01-286r

Wachter-Boettcher, S. (2017). Technically wrong: Sexist apps, biased algorithms, and other threats of toxic tech. WW Norton \& Company.

Weiser, M. (1991, September). The computer of the 21st century. Scientific American, 265(3), 94-105.

West, S. M., Whittaker, M., \& Crawford, K. (2019). Discriminating systems. Al Now Institute. https://cdn. vox-cdn.com/uploads/chorus_asset/file/16125391/ discriminating_systems_041519_2.pdf

Whittaker, M., Crawford, K., Dobbe, R., Fried, G., Kaziunas, E., Mathur, V., Mysers West, S., Richardson, R., Schultz, J., \& Schwartz, O. (2018). Al now report 2018. Al Now Institute at New York University New York.

Zuboff, S. (2015). Big other: surveillance capitalism and the prospects of an information civilization. Journal of Information Technology, 30(1), 75-89. https://doi. org/10.1057/jit.2015.5

Zweben, S., \& Bizot, B. (2020). 2020 Taulbee Survey. Computing Research Association (CRA). https://cra. org/wp-content/uploads/2021/05/2020-CRA-TaulbeeSurvey.pdf 